\title{
Bird communities in two fragments of Cerrado in Itirapina, Brazil
}

\author{
Telles, M.* and Dias, MM. \\ Departamento de Ecologia e Biologia Evolutiva, Universidade Federal de São Carlos - UFSCar, \\ Rodovia Washington Luís, Km 235, CEP 13565-905, São Carlos, SP, Brazil \\ *e-mail: marina.telles@gmail.com
}

Received April 1, 2009 - Accepted June 18, 2009 - Distributed August 31, 2010

(With 4 figures)

\begin{abstract}
The Cerrado domain is a mosaic of vegetation types at the local scale, and this environmental heterogeneity leads to high regional bird diversity. Therefore, we aimed to survey quantitative and qualitatively the bird fauna of two fragments of Cerrado and to compare them with an adjacent protected area (Estação Ecológica de Itirapina), in order to assess the heterogeneity of bird diversity in the region. The present study was conducted during 12 months from October 2006 to September 2007 in the municipality of Itirapina, Southeastern Brazil. Altogether we recorded 210 bird species. Fifty-six of them had never been detected in Estação Ecológica de Itirapina, and eleven species are new records for the whole Itirapina region. The list also includes six species that are endangered in Sao Paulo State and five endemic species of the Cerrado domain. Most species were recorded in less than $50 \%$ of the visits and exhibited low relative abundance. Primarily insectivorous species were the most common, followed by omnivores. Frugivorous birds were poorly represented. Carnivores were more abundant than usually observed in fragments. The similarity among fragments was higher than between fragments and the protected area. Considering the vegetation heterogeneity in the Cerrado domain, our results reinforce the importance of conserving fragments in order to sample this diversity.
\end{abstract}

Keywords: avifauna, forest fragmentation, environmental heterogeneity.

\section{Comunidades de aves em dois fragmentos de Cerrado em Itirapina, Brasil}

\section{Resumo}

O mosaico de tipos vegetacionais que formam o Cerrado contribui para a alta diversidade de aves deste domínio. O presente trabalho teve como objetivo principal realizar os levantamentos qualitativo e quantitativo da avifauna de dois fragmentos de Cerrado e compará-los aos resultados encontrados em uma área adjacente de Cerrado (Estação Ecológica de Itirapina). O trabalho foi realizado entre outubro de 2006 e setembro de 2007, no município de Itirapina, região central do Estado de São Paulo, Brasil. Registramos um total de 210 espécies de aves. Destas, 56 ainda não haviam sido registradas na Estação Ecológica e onze espécies são novos registros para a região de Itirapina. A lista dos fragmentos inclui seis espécies ameaçadas no Estado de São Paulo e cinco espécies endêmicas de Cerrado. A maioria das espécies foi detectada em menos de $50 \%$ das visitas e apresentou baixa abundância relativa. A classificação em categorias tróficas indicou a prevalência de espécies predominantemente insetívoras, seguidas pelas onívoras. Os frugívoros foram pouco representados e a quantidade de carnívoros foi superior à geralmente encontrada em fragmentos. A similaridade encontrada entre os fragmentos foi maior que a encontrada entre os fragmentos e Estação Ecológica. Os resultados reforçam a importância da conservação dos fragmentos de Cerrado, uma vez que a heterogeneidade de tipos vegetacionais é importante para a conservação da biodiversidade local.

Palavras-chave: avifauna, fragmentação florestal, heterogeneidade ambiental.

\section{Introduction}

Detecting environmental quality and monitoring biodiversity is important for the conservation of natural areas. In that sense, bird inventories are commonly used for this purpose (Vielliard, 2000; Marini, 2001; Antunes, 2005; Piratelli et al., 2008). Conservation strategies of natural areas require knowledge on the distribution and abundance of species, and studies on bird communities can provide suitable data (Naeve et al., 1996).
Studies on Cerrado demonstrate high biodiversity (Klink and Machado, 2005). The domain is formed of a mosaic of different vegetation forms that varies from grasslands ('campo limpo') to forests ('cerradão'), including gallery forests (Coutinho, 2006). This environmental heterogeneity is important to keep high levels of species richness and endemic species (Machado et al., 2004). However, the Cerrado is one of the world's threatened biodiversity 
hotspots (Myers et al., 2000). About $60 \%$ of its vegetation has already been removed (Machado et al., 2004) and the remaining areas are isolated in forest fragments (Durigan et al., 2007).

Nowadays, Sao Paulo State has only $7 \%$ of its original Cerrado vegetation (Duringan et al., 2007), and only $18 \%$ is inside protected areas (São Paulo, 1999). The Estação Ecológica de Itirapina (EEI) is one of these areas and has been studied by ornithologists since the 80's (Willis, 2004). It has already 231 bird species registered (Willis, 2004; Motta-Junior et al., 2008), including endemic and threatened ones (Silva and Bates, 2002; IBAMA, 2008; IUCN, 2008; São Paulo, 2008). That makes the area important for bird biodiversity conservation (Bencke et al., 2006). Close to the EEI there is another protected area, the Estação Experimental de Itirapina, which has two fragments of Cerrado poorly studied in terms of its avifauna (Willis and Oniki, 2003).

While grasslands are predominant in EEI (Motta-Junior et al., 2008; Granzinolli, 2009) more forested areas prevail in these two fragments. Therefore, this paper aimed to accomplish qualitative and quantitative bird surveys of the two fragments, as well as to compare the results with those from EEI. Such outcomes could be used in future wildlife monitoring and to better understand the influence of fragmentation on bird communities (Palmer et al., 2008). This study also proposes some local management strategies for biodiversity conservation.

\section{Material and Methods}

\subsection{Study area}

We have studied two fragments: A $\left(22^{\circ} 13^{\prime} \mathrm{S}\right.$ and $\left.47^{\circ} 48^{\prime} \mathrm{W}\right)$ and $\mathrm{B}\left(22^{\circ} 14^{\prime} \mathrm{S}\right.$ and $\left.47^{\circ} 49^{\prime} \mathrm{W}\right)$, inside the Estação Experimental de Itirapina. This area is basically used for the extraction of Eucalyptus spp. (Myrtaceae) and resin from Pinus spp. (Pinacea). Fragment A, of 150 ha, is composed predominantly of 'cerrado sensu stricto', 'cerradão' (a tall woodland) and gallery forest (Delgado et al., 2004). Fragment B, of 350 ha, is more heterogeneous, concerning the vegetation. It is a more forested fragment than A, being composed of 'cerradão', 'cerrado sensu stricto', gallery forests (Delgado et al., 2004), Eucalyptus spp. with native understory and regeneration areas with native and exotic species (pers. obs.). Fragments A and B are about $1.5 \mathrm{~km}$ and $2 \mathrm{~km}$, respectively, distant from EEI, that is of 2,300 ha. Both fragments are surrounded by pine and eucalyptus plantations and are $750 \mathrm{~m}$ apart from each other.

Itirapina region has a Cwa climate according to the Köppen system (Zanchetta, 2006), with annual rainfall average of $1,459 \mathrm{~mm}$. The rainy season is usually from October to March and the dry period occurs from April to September. The average annual temperature is $21.9^{\circ} \mathrm{C}$. The hottest months are January and February and the coolest, June and July. The altitude of the region is about $740 \mathrm{~m}$ (Zanchetta, 2006).

\subsection{Methods}

Qualitative and quantitative methods were performed. The qualitative survey was done to generate the fullest species list possible that occur in the area (Vielliard and Silva, 1990) and was conducted during 12 months (October 2006 to September 2007). For this purpose we walked on pre-existent tracks during mornings (6:00 to 11:00 AM), evenings (3:00 to 6:00 PM) and nights (6:00 to 9:00 PM) recording all bird species, through auditory and/or visual contacts, without standardising the vegetation types. Sampling effort during the qualitative survey in fragment $\mathrm{B}$ (61 visits) was higher than in fragment A (30 visits) because of its size and vegetation heterogeneity. Rarefaction curves were plotted using the statistical package EstimateS 8.0 (Colwell, 2006). The same program was used to estimate fragment richness using the first-order Jackknife richness estimator (Heltshe and Forrester, 1983). Species composition in the fragments, compared with that from EEI, was analysed using Jaccard's similarity index (Krebs, 1999). Bird lists from EEI were extracted from data review by Motta-Junior et al. (2008). Species frequency of occurrence (FO) was calculated from Vielliard and Silva (1990) and the analysis of trophic structure was done using species groups based on Motta-Junior (1990) and Sick (1997).

The quantitative survey was also carried out over 12 months (October 2006 to September 2007) using the point counts method, developed by Blondel et al. (1970) and adapted by Vielliard and Silva (1990) for Neotropical studies. For this purpose, 18 sampling points were chosen in each fragment in pre-existing tracks, each containing four or five points, with the minimum distance of $200 \mathrm{~m}$ from each other (Vielliard and Silva, 1990). In each of these sampling places all auditory and/or visual contacts with individuals within a $100 \mathrm{~m}$ range were recorded over 20 minutes. Points were visited during morning (6:00 to 11:00 PM), starting minutes before sunrise. On each day, eight to ten random points were visited in each fragment, totalising 216 samples in each area (18 points visited for 12 months).

Through quantitative surveys the Ponctual Index of Abundance (PIA) was calculated (Vielliard and Silva, 1990; Aleixo and Vielliard, 1995). The Shannon diversity index (H') and Pielou's evenness (J') (Krebs, 1999) were calculated using PAST statistical package (Hammer et al., 2001). We used Bootstrap ( $\mathrm{p}<0.05)$ to reduce bias (Hammer et al., 2001). Scientific nomenclature adopted here follows the Brazilian Committee of Ornithological Records (CBRO, 2008).

\section{Results}

During the qualitative survey, 210 species of birds distributed in 51 families, were recorded (see Table 1). In 91 days of field visits (476 hours of observation) 158 species in the fragment A and 201 in B were recorded (see Table 1). First-order Jackknife estimated a richness of $186 \pm 7.22$ species to fragment $A$ and $223.61 \pm 5.3$ species 
to fragment B. Rarefaction curves from both fragments indicate a similar pattern (see Figures 1 and 2). The similarity among species composition of fragments was $70.95 \%$, while the similarity between fragments and EEI was $53.66 \%$.

Species frequency of occurrence ranged from $3.1 \%$ (one contact) to $96.9 \%$ (31 contacts) in fragment $\mathrm{A}$ and $1.5 \%$ (one contact) to $90.8 \%$ (59 contacts) in B (see Table 1). In both fragments, most of the species were recorded in up to $50 \%$ of the field visits (see Table 2), namely 119 species in A (75.3\% of the total recorded in the fragment) and 158 species in B $(78.61 \%)$, however some species were recorded in only one visit. This occurred with 30 species $(18.9 \%)$ in A and $22(13.92 \%)$ in B. Species with frequency of occurrence over $75 \%$ were considered residents in the studied areas (Almeida et al., 1999). This frequency was 19 species (12.03\%) for fragment A and 14 species (6.97\%) for B (see Table 1).

In both fragments, results were similar in relation to the distribution of trophic categories (see Table 3). Predominantly insectivorous birds were more representative both in A and B ( 49.37 and $48.26 \%$ respectively), followed by predominantly omnivorous (17.72 and $16.42 \%$, respectively). The other categories, in order of prevalence piscivores, detritivores, malacophages, carnivores, nectarivores and gramnivores - were poorly represented and together summed only $27.21 \%$ of birds in fragment A and $29.36 \%$ in B. The predominantly frugivorous birds were represented by only $5.70 \%$ of species in A and $5.97 \%$ in B.

During quantitative surveys, 111 species of birds in A and 120 in B were recorded, in 3,647 and 3,474 contacts, respectively (see Table 1). PIA ranged from 0.005 (one contact) to 1.282 (277 contacts) in A and 0.005 (one contact) to 1.023 (221 contacts) in B (see Figures 3 and 4). In A, 26 species ( $23.42 \%$ of the total registered during quantitative survey) had PIA values lower than 0.019 (less than four contacts), and the same occurred with 36 species $(29.75 \%$ ) in $\mathrm{B}$, where values lower than 0.014 represent less than three contacts.

The Shannon diversity index was H' $=3.9$ for both fragments and Pielou's evenness was J' $=0.85$ for fragment A and J' $=0.84$ for fragment B. Data indicate high similarity in community structure among fragments.

Table 1. Bird species recorded during qualitative and quantitative surveys in two Brazilian Cerrado fragments in Itirapina, Sao Paulo. PIA - Ponctual Index of Abundance, FO - Frequency of Occurrence given in percentage, A (fragment A), B (fragment B). The nine trophic categories are represented by: ONI (omnivores), FRU (frugivores), PIS (piscivores), INS (insectivores), DET (detritivores), CAR (carnivores), MAL (malacophages), GRA (gramnivores) and NEC (nectarivores) - based on Motta-Junior (1990) and Sick (1997).

\begin{tabular}{|c|c|c|c|c|c|}
\hline \multirow[t]{2}{*}{ Taxon } & \multicolumn{2}{|c|}{ FO $(\%)$} & \multicolumn{2}{|c|}{ PIA } & \multirow{2}{*}{$\begin{array}{c}\text { Trophic } \\
\text { categories }\end{array}$} \\
\hline & $\mathbf{A}$ & B & $\mathbf{A}$ & B & \\
\hline \multicolumn{6}{|l|}{ TINAMIDAE } \\
\hline Crypturellus parvirostris (Wagler, 1827) & 46.9 & 33.8 & 0.111 & 0.054 & ONI \\
\hline \multicolumn{6}{|l|}{ ANATIDAE } \\
\hline Amazonetta brasiliensis (Gmelin, 1789) & - & 41.5 & - & - & ONI \\
\hline \multicolumn{6}{|l|}{ CRACIDAE } \\
\hline Penelope superciliaris Temminck, 1815 & 25 & 16.9 & 0.019 & 0.037 & FRU \\
\hline \multicolumn{6}{|l|}{ PHALACROCORACIDAE } \\
\hline Phalacrocorax brasilianus (Gmelin, 1789) & 3.1 & 46.2 & - & - & PIS \\
\hline \multicolumn{6}{|l|}{ ARDEIDAE } \\
\hline Tigrisoma lineatum (Boddaert, 1783) & - & 9.2 & - & - & PIS \\
\hline Nycticorax nycticorax (Linnaeus, 1758) * & 3.1 & 7.7 & - & - & PIS \\
\hline Butorides striata (Linnaeus, 1758) & - & 3.1 & - & - & PIS \\
\hline Bubulcus ibis (Linnaeus, 1758) & 25 & 23.1 & - & - & PIS \\
\hline Ardea cocoi Linnaeus, 1766 & 15.6 & 33.8 & - & - & PIS \\
\hline Ardea alba Linnaeus, 1758 & 18.8 & 66.2 & - & - & PIS \\
\hline Syrigma sibilatrix (Temminck, 1824) & 37.5 & 6.2 & - & - & PIS \\
\hline Egretta thula (Molina, 1782) & 3.1 & 49.2 & - & - & PIS \\
\hline \multicolumn{6}{|l|}{ THRESKIORNITHIDAE } \\
\hline Mesembrinibis cayennensis (Gmelin, 1789) & 12.5 & 33.8 & - & 0.023 & INS \\
\hline Theristicus caudatus (Boddaert, 1783) & - & 1.5 & - & - & INS \\
\hline \multicolumn{6}{|l|}{ CATHARTIDAE } \\
\hline Cathartes aura (Linnaeus, 1758) & - & 3.1 & - & - & DET \\
\hline Coragyps atratus (Bechstein, 1793) & 43.8 & 47.7 & - & - & DET \\
\hline
\end{tabular}

* Species that had not been recorded in EEI $(n=56)$ (review by Motta-Junior et al., 2008); $§$ Atlantic Forest endemic species $(n=16)$ (Brooks et al., 1999); $\uparrow$ Species registered for the first time for the whole Itirapina region $(\mathrm{n}=11)$ (Willis, 2003a; Willis and Oniki 2003; Motta-Junior et al., 2008); and $\ddagger$ Fragments resident species $(\mathrm{n}=23)$ (Almeida et al., 1999). 
Table 1. Continued...

\begin{tabular}{|c|c|c|c|c|c|}
\hline \multirow[t]{2}{*}{ Taxon } & \multicolumn{2}{|c|}{ FO $(\%)$} & \multicolumn{2}{|c|}{ PIA } & \multirow{2}{*}{$\begin{array}{c}\text { Trophic } \\
\text { categories }\end{array}$} \\
\hline & $\mathbf{A}$ & B & A & B & \\
\hline \multicolumn{6}{|l|}{ ACCIPITRIDAE } \\
\hline Elanus leucurus (Vieillot, 1818) & 3.1 & 1.5 & - & - & CAR \\
\hline Rostramus sociabilis (Vieillot, 1817) $* \dagger$ & 6.3 & 3.1 & - & - & MAL \\
\hline Ictinia plumbea (Gmelin, 1788) & 21.9 & 30.8 & - & - & INS \\
\hline Geranospiza caerulescens (Vieillot, 1817) * & - & 13.8 & - & - & CAR \\
\hline Buteogallus urubitinga (Gmelin, 1788) & 6.3 & - & - & - & CAR \\
\hline Heterospizias meridionalis (Latham, 1790) & - & 1.5 & - & - & CAR \\
\hline Rupornis magnirostris (Gmelin, 1788) $\ddagger$ & 81.3 & 69.2 & - & - & CAR \\
\hline Buteo albicaudatus Vieillot, 1816 & 3.1 & 10.8 & - & - & CAR \\
\hline Buteo brachyurus Vieillot, 1816 * & 3.1 & 13.8 & - & - & CAR \\
\hline \multicolumn{6}{|l|}{ FALCONIDAE } \\
\hline Caracara plancus (Miller, 1777) & 71.9 & 40 & - & - & CAR \\
\hline Milvago chimachima (Vieillot, 1816) & 43.8 & 38.5 & - & - & CAR \\
\hline Herpetotheres cachinnans (Linnaeus, 1758) & 9.4 & 4.6 & - & - & CAR \\
\hline Micrastur semitorquatus (Vieillot, 1817) $* \dagger$ & - & 13.8 & - & - & CAR \\
\hline Falco sparverius Linnaeus, 1758 & - & 3.1 & - & - & INS \\
\hline Falco femoralis Temminck, 1822 & 6.3 & 6.2 & - & - & CAR \\
\hline \multicolumn{6}{|l|}{ RALLIDAE } \\
\hline Aramides cajanea (Statius Muller, 1776) & - & 4.6 & - & - & ONI \\
\hline \multicolumn{6}{|l|}{ CARIAMIDAE } \\
\hline Cariama cristata (Linnaeus, 1766) $\ddagger$ & 84.4 & 61.5 & - & - & INS \\
\hline \multicolumn{6}{|l|}{ CHARADRIIDAE } \\
\hline Vanellus chilensis (Molina, 1782) & 68.8 & 70.8 & - & - & ONI \\
\hline \multicolumn{6}{|l|}{ SCOLOPACIDAE } \\
\hline Tringa solitaria Wilson, 1813 * & - & 3.1 & - & - & INS \\
\hline \multicolumn{6}{|l|}{ COLUMBIDAE } \\
\hline Columbina talpacoti (Temminck, 1811) & 46.9 & 33.8 & 0.125 & 0.046 & GRA \\
\hline Columbina squammata (Lesson, 1831) & 53.1 & 33.8 & 0.144 & 0.079 & GRA \\
\hline Patagioenas picazuro $($ Temminck, 1813$) \ddagger$ & 96.9 & 89.2 & 1.282 & 1.023 & FRU \\
\hline Patagioenas cayennensis (Bonnaterre, 1792) & 53.1 & 36.9 & 0.125 & 0.069 & FRU \\
\hline Zenaida auriculata (Des Murs, 1847) & 9.4 & 9.2 & 0.005 & - & GRA \\
\hline Leptotila verreauxi Bonaparte, $1855 \ddagger$ & 84.4 & 70.8 & 0.444 & 0.375 & FRU \\
\hline Leptotila rufaxilla (Richard \& Bernard, 1792) $* \dagger$ & - & 3.1 & - & - & FRU \\
\hline \multicolumn{6}{|l|}{ PSITTACIDAE } \\
\hline Aratinga leucophthalma (Statius Muller, 1776) & 68.8 & 58.5 & - & - & FRU \\
\hline Aratinga aurea (Gmelin, 1788) & - & 1.5 & - & - & FRU \\
\hline Forpus xanthopterygius (Spix, 1824) & 25 & 52.3 & - & - & FRU \\
\hline Brotogeris chiriri (Vieillot, 1818) & 65.6 & 52.3 & - & - & FRU \\
\hline \multicolumn{6}{|l|}{ CUCULIDAE } \\
\hline Piaya cayana (Linnaeus, 1766) & 34.4 & 40 & 0.028 & 0.065 & INS \\
\hline Crotophaga ani Linnaeus, 1758 & 31.3 & 27.7 & 0.037 & 0.037 & INS \\
\hline Guira guira (Gmelin, 1788) & 3.1 & 3.1 & 0.005 & - & INS \\
\hline Tapera naevia (Linnaeus, 1766) & 9.4 & 13.8 & 0.009 & 0.009 & INS \\
\hline \multicolumn{6}{|l|}{ TYTONIDAE } \\
\hline Tyto alba (Scopoli, 1769) & - & 1.5 & - & - & CAR \\
\hline \multicolumn{6}{|l|}{ STRIGIDAE } \\
\hline Glaucidium brasilianum (Gmelin, 1788) & - & 1.5 & - & - & CAR \\
\hline Athene cunicularia (Molina, 1782) & - & 1.5 & - & - & CAR \\
\hline Rhinoptynx clamator (Vieillot, 1808) & - & 4.6 & - & - & CAR \\
\hline
\end{tabular}

* Species that had not been recorded in EEI $(n=56)$ (review by Motta-Junior et al., 2008); § Atlantic Forest endemic species $(n=16)$ (Brooks et al., 1999); $\uparrow$ Species registered for the first time for the whole Itirapina region $(n=11)$ (Willis, 2003a; Willis and Oniki 2003; Motta-Junior et al., 2008); and $\ddagger$ Fragments resident species $(\mathrm{n}=23)$ (Almeida et al., 1999). 
Table 1. Continued...

\begin{tabular}{|c|c|c|c|c|c|}
\hline \multirow[t]{2}{*}{ Taxon } & \multicolumn{2}{|c|}{ FO $(\%)$} & \multicolumn{2}{|c|}{ PIA } & \multirow{2}{*}{$\begin{array}{c}\text { Trophic } \\
\text { categories }\end{array}$} \\
\hline & $\mathbf{A}$ & B & A & B & \\
\hline Asio stygius (Wagler, 1832) & - & 3.1 & - & - & CAR \\
\hline \multicolumn{6}{|l|}{ NYCTIBIIDAE } \\
\hline Nyctibius griseus (Gmelin, 1789) & 3.1 & 9.2 & - & - & INS \\
\hline \multicolumn{6}{|l|}{ CAPRIMULGIDAE } \\
\hline Podager nacunda (Vieillot, 1817) * & - & 1.5 & - & - & INS \\
\hline Nyctidromus albicollis (Gmelin, 1789) & 50 & 56.9 & - & - & INS \\
\hline Caprimulgus rufus Boddaert, $1783 *$ & 6.3 & 15.4 & - & - & INS \\
\hline Hydropsalis torquata (Gmelin, 1789) & 12.5 & 9.2 & - & - & INS \\
\hline \multicolumn{6}{|l|}{ APODIDAE } \\
\hline Streptoprocne zonaris (Shaw, 1796) & - & 1.5 & - & - & INS \\
\hline Chaetura meridionalis Hellmayr, 1907 & 6.3 & 3.1 & - & - & INS \\
\hline \multicolumn{6}{|l|}{ TROCHILIDAE } \\
\hline Phaethornis pretrei (Lesson \& Delattre, 1839) & 9.4 & 9.2 & - & 0.019 & NEC \\
\hline Eupetomena macroura (Gmelin, 1788) & 25 & 23.1 & 0.023 & 0.032 & NEC \\
\hline Aphantochroa cirrochloris (Vieillot, 1818) § & 3.1 & 10.8 & 0.005 & 0.009 & NEC \\
\hline Florisuga fusca (Vieillot, 1817) $§$ & 46.9 & 36.9 & 0.111 & 0.111 & NEC \\
\hline Anthracothorax nigricollis (Vieillot, 1817) * & - & 1.5 & 0.130 & - & NEC \\
\hline Chlorostilbon lucidus (Shaw, 1812) & 34.4 & 47.7 & - & 0.111 & NEC \\
\hline Thalurania glaucopis (Gmelin, 1788) * $\S$ & - & 1.5 & - & - & NEC \\
\hline Hylocharis sapphirina (Gmelin, 1788) $* \dagger$ & 6.3 & 3.1 & 0.014 & 0.005 & NEC \\
\hline Hylocharis chrysura (Shaw, 1812) & 46.9 & 43.1 & 0.162 & 0.120 & NEC \\
\hline Leucochloris albicollis (Vieillot, 1818) $* \S$ & 12.5 & 9.2 & 0.023 & 0.005 & NEC \\
\hline Amazilia versicolor (Vieillot, 1818) & 3.1 & 3.1 & 0.005 & 0.005 & NEC \\
\hline Amazilia lactea (Lesson, 1832) & 56.3 & 60 & 0.278 & 0.130 & NEC \\
\hline Heliomaster squamosus (Temminck, 1823) * & 34.4 & 26.2 & 0.065 & 0.023 & NEC \\
\hline Calliphlox amethystina (Boddaert, 1783) * & - & 1.5 & - & 0.005 & NEC \\
\hline \multicolumn{6}{|l|}{ ALCEDINIDAE } \\
\hline Megaceryle torquata (Linnaeus, 1766) & 3.1 & 29.2 & 0.005 & 0.032 & PIS \\
\hline Chloroceryle amazona (Latham, 1790) & 3.1 & 50.8 & 0.005 & 0.005 & PIS \\
\hline \multicolumn{6}{|l|}{ MOMOTIDAE } \\
\hline Baryphthengus ruficapillus (Vieillot, 1818)* $§$ & 9.4 & 43.1 & 0.014 & 0.088 & ONI \\
\hline \multicolumn{6}{|l|}{ GALBULIDAE } \\
\hline Galbula ruficauda Cuvier, 1816 & 3.1 & - & - & - & INS \\
\hline \multicolumn{6}{|l|}{ BUCCONIDAE } \\
\hline Nystalus chacuru (Vieillot, 1816) & - & 1.5 & - & 0.005 & INS \\
\hline Malacoptila striata (Spix, 1824) * $§$ & - & 1.5 & - & 0.005 & INS \\
\hline \multicolumn{6}{|l|}{ RAMPHASTIDAE } \\
\hline Ramphastos toco Statius Muller, 1776 & - & 21.5 & - & 0.014 & FRU \\
\hline \multicolumn{6}{|l|}{ PICIDAE } \\
\hline Picumnus albosquamatus d'Orbigny, 1840 & 59.4 & 69.2 & 0.144 & 0.185 & INS \\
\hline Melanerpes candidus (Otto, 1796) & 18.8 & 15.4 & 0.051 & 0.056 & INS \\
\hline Veniliornis passerinus (Linnaeus, 1766) & 43.8 & 41.5 & 0.097 & 0.069 & INS \\
\hline Colaptes melanochloros (Gmelin, 1788) & 53.1 & 47.7 & 0.106 & 0.097 & INS \\
\hline Colaptes campestris (Vieillot, 1818) $\ddagger$ & 84.4 & 49.2 & 0.255 & 0.088 & INS \\
\hline Dryocopus lineatus (Linnaeus, 1766) & 50 & 38.5 & 0.106 & 0.032 & INS \\
\hline \multicolumn{6}{|l|}{ MELANOPAREIIDAE } \\
\hline Melanopareia torquata (Wied, 1831) & - & 1.5 & - & - & INS \\
\hline \multicolumn{6}{|l|}{ THAMNOPHILIDAE } \\
\hline Taraba major (Vieillot, 1816) & 15.6 & 7.7 & 0.032 & - & INS \\
\hline
\end{tabular}

* Species that had not been recorded in EEI $(n=56)$ (review by Motta-Junior et al., 2008); $§$ Atlantic Forest endemic species $(n=16)$ (Brooks et al., 1999); $\dagger$ Species registered for the first time for the whole Itirapina region $(n=11)$ (Willis, 2003a; Willis and Oniki 2003; Motta-Junior et al., 2008); and $\ddagger$ Fragments resident species $(\mathrm{n}=23)$ (Almeida et al., 1999). 
Table 1. Continued...

\begin{tabular}{|c|c|c|c|c|c|}
\hline \multirow[t]{2}{*}{ Taxon } & \multicolumn{2}{|c|}{ FO $(\%)$} & \multicolumn{2}{|c|}{ PIA } & \multirow{2}{*}{$\begin{array}{c}\text { Trophic } \\
\text { categories }\end{array}$} \\
\hline & $\mathbf{A}$ & B & A & B & \\
\hline Thamnophilus doliatus (Linnaeus, 1764) & 34.4 & 12.3 & 0.065 & 0.014 & INS \\
\hline Thamnophilus ruficapillus Vieillot, 1816 & - & 3.1 & - & 0.009 & INS \\
\hline Thamnophilus torquatus Swainson, 1825 & - & 1.5 & - & - & INS \\
\hline Thamnophilus pelzelni Hellmayr, $1924 * \ddagger$ & 81.3 & 47.7 & 0.361 & 0.204 & INS \\
\hline Thamnophilus caerulescens Vieillot, 1816 & 50 & 66.2 & 0.083 & 0.222 & INS \\
\hline Dysithamnus mentalis (Temminck, 1823) * & 3.1 & 4.6 & - & 0.005 & INS \\
\hline Formicivora rufa (Wied, 1831) & 28.1 & 4.6 & 0.097 & 0.005 & INS \\
\hline Drymophila ferruginea (Temminck, 1822) * $\S$ & 6.3 & 4.6 & - & - & INS \\
\hline \multicolumn{6}{|l|}{ CONOPOPHAGIDAE } \\
\hline Conopophaga lineata (Wied, 1831) § & 34.4 & 55.4 & 0.065 & 0.134 & INS \\
\hline \multicolumn{6}{|l|}{ DENDROCOLAPTIDAE } \\
\hline Sittasomus griseicapillus (Vieillot, 1818) * & 9.4 & 12.3 & 0.014 & 0.023 & INS \\
\hline Lepidocolaptes angustirostris (Vieillot, 1818) & 62.5 & 47.7 & 0.231 & 0.079 & INS \\
\hline \multicolumn{6}{|l|}{ FURNARIIDAE } \\
\hline Furnarius rufus (Gmelin, 1788) & 31.3 & 41.5 & 0.046 & 0.079 & INS \\
\hline Synallaxis ruficapilla Vieillot, $1819 * \S$ & - & 4.6 & - & - & INS \\
\hline Synallaxis frontalis Pelzeln, $1859 \ddagger$ & 81.3 & 64.6 & 0.190 & 0.083 & INS \\
\hline Synallaxis albescens Temminck, 1823 & 3.1 & - & - & - & INS \\
\hline Synallaxis spixi Sclater, 1856 & 40.6 & 66.2 & 0.069 & 0.116 & INS \\
\hline Certhiaxis cinnamomeus (Gmelin, 1788) & - & 27.7 & - & - & INS \\
\hline Phacellodomus ferrugineigula (Pelzeln, 1858) § & 6.3 & 3.1 & 0.005 & - & INS \\
\hline Automolus leucophthalmus (Wied, 1821) * & 12.5 & 41.5 & 0.005 & 0.079 & INS \\
\hline Xenops rutilans Temminck, $1821 *$ & 6.3 & 6.2 & 0.005 & - & INS \\
\hline \multicolumn{6}{|l|}{ TYRANNIDAE } \\
\hline Leptopogon amaurocephalus Tschudi, 1846 * & 6.3 & 30.8 & - & 0.074 & INS \\
\hline Hemitriccus orbitatus (Wied, 1831) $* \S$ & - & 1.5 & - & - & INS \\
\hline Hemitriccus margaritaceiventer (d'Orbigny \& Lafresnaye, 1837) & 21.9 & - & 0.023 & 0.005 & INS \\
\hline Todirostrum poliocephalum (Wied, 1831) * $\S$ & - & 1.5 & - & - & INS \\
\hline Todirostrum cinereum (Linnaeus, 1766) & - & 1.5 & - & 0.005 & INS \\
\hline Elaenia flavogaster (Thunberg, 1822) & 71.9 & 66.2 & 0.579 & 0.282 & ONI \\
\hline Elaenia spectabilis Pelzeln, $1868 * \dagger$ & 3.1 & 6.2 & 0.019 & - & ONI \\
\hline Elaenia mesoleuca (Deppe, 1830) & 18.8 & 7.7 & 0.037 & 0.037 & ONI \\
\hline Elaenia chiriquensis Lawrence, 1865 & 34.4 & 24.6 & 0.153 & 0.093 & ONI \\
\hline Elaenia obscura (d'Orbigny \& Lafresnaye, 1837) & 43.8 & 43.1 & 0.167 & 0.120 & ONI \\
\hline Camptostoma obsoletum (Temminck, 1824) $\ddagger$ & 87.5 & 81.5 & 0.444 & 0.287 & INS \\
\hline Serpophaga nigricans (Vieillot, 1817) & 3.1 & - & - & - & INS \\
\hline Serpophaga subcristata (Vieillot, 1817) & 34.4 & 26.2 & 0.125 & 0.037 & INS \\
\hline Phaeomyias murina (Spix, 1825) * & 31.3 & 16.9 & 0.106 & 0.056 & INS \\
\hline Euscarthmus meloryphus Wied, $1831 *$ & 21.9 & 29.2 & 0.046 & 0.028 & INS \\
\hline Tolmomyias sulphurescens (Spix, 1825) $* \ddagger$ & 40.6 & 83.1 & 0.069 & 0.306 & INS \\
\hline Platyrinchus mystaceus Vieillot, $1818 *$ & 3.1 & 6.2 & - & - & INS \\
\hline Myiophobus fasciatus (Statius Muller, 1776) & 6.3 & 10.8 & - & 0.009 & INS \\
\hline Lathrotriccus euleri (Cabanis, 1868) & 34.4 & 46.2 & 0.037 & 0.116 & INS \\
\hline Cnemotriccus fuscatus (Wied, 1831) * & 40.6 & 24.6 & 0.102 & 0.051 & INS \\
\hline Knipolegus cyanirostris (Vieillot, 1818) * & - & 9.2 & - & 0.014 & INS \\
\hline Satrapa icterophrys (Vieillot, 1818) & 3.1 & 4.6 & 0.009 & - & INS \\
\hline Xolmis velatus (Lichtenstein, 1823) & 3.1 & - & 0.009 & - & INS \\
\hline Fluvicola nengeta (Linnaeus, 1766) * & - & 32.3 & - & - & INS \\
\hline Colonia colonus (Vieillot, 1818) * & 25 & 33.8 & 0.028 & 0.037 & INS \\
\hline
\end{tabular}

* Species that had not been recorded in EEI $(\mathrm{n}=56$ ) (review by Motta-Junior et al., 2008); $§$ Atlantic Forest endemic species $(\mathrm{n}=16)$ (Brooks et al., 1999); $\uparrow$ Species registered for the first time for the whole Itirapina region $(n=11)$ (Willis, 2003a; Willis and Oniki 2003; Motta-Junior et al., 2008); and $\ddagger$ Fragments resident species $(\mathrm{n}=23)$ (Almeida et al., 1999). 
Table 1. Continued...

\begin{tabular}{|c|c|c|c|c|c|}
\hline \multirow[t]{2}{*}{ Taxon } & \multicolumn{2}{|c|}{ FO $(\%)$} & \multicolumn{2}{|c|}{ PIA } & \multirow{2}{*}{$\begin{array}{l}\text { Trophic } \\
\text { categories }\end{array}$} \\
\hline & $\mathbf{A}$ & B & A & B & \\
\hline Machetornis rixosa (Vieillot, 1819) & - & 1.5 & - & - & INS \\
\hline Legatus leucophaius (Vieillot, 1818) $* \dagger$ & 9.4 & 13.8 & 0.005 & 0.028 & INS \\
\hline Myiozetetes similis (Spix, 1825) & 65.6 & 61.5 & 0.134 & 0.116 & ONI \\
\hline Pitangus sulphuratus (Linnaeus, 1766) $\ddagger$ & 84.4 & 90.8 & 0.634 & 0.556 & ONI \\
\hline Myiodynastes maculatus (Statius Muller, 1776) & 34.4 & 38.5 & 0.153 & 0.231 & ONI \\
\hline Megarynchus pitangua (Linnaeus, 1766) $\ddagger$ & 84.4 & 86.2 & 0.329 & 0.370 & ONI \\
\hline Empidonomus varius (Vieillot, 1818) & 40.6 & 44.6 & 0.120 & 0.167 & INS \\
\hline Tyrannus albogularis Burmeister, 1856 & 9.4 & 3.1 & - & - & INS \\
\hline Tyrannus melancholicus Vieillot, $1819 \ddagger$ & 78.1 & 61.5 & 0.759 & 0.454 & INS \\
\hline Tyrannus savana Vieillot, 1808 & 28.1 & 32.3 & 0.153 & 0.037 & INS \\
\hline Casiornis rufus (Vieillot, 1816)* & 62.5 & 35.4 & 0.264 & 0.056 & INS \\
\hline Myiarchus swainsoni Cabanis \& Heine, 1859 & 31.3 & 26.2 & 0.037 & 0.014 & INS \\
\hline Myiarchus ferox (Gmelin, 1789) & 68.8 & 46.2 & 0.241 & 0.093 & INS \\
\hline Myiarchus tyrannulus (Statius Muller, 1776) & 18.8 & 3.1 & 0.023 & - & INS \\
\hline \multicolumn{6}{|l|}{ PIPRIDAE } \\
\hline Antilophia galeata (Lichtenstein, 1823) & 40.6 & 44.6 & 0.069 & 0.069 & FRU \\
\hline Chiroxiphia caudata (Shaw \& Nodder, 1793) * $§$ & 3.1 & 36.9 & - & 0.065 & FRU \\
\hline \multicolumn{6}{|l|}{ TITYRIDAE } \\
\hline Pachyramphus castaneus (Jardine \& Selby, 1827) $* \dagger$ & - & 1.5 & - & - & INS \\
\hline Pachyramphus polychopterus (Vieillot, 1818)* & 40.6 & 36.9 & 0.125 & 0.093 & INS \\
\hline Pachyramphus validus (Lichtenstein, 1823) * & 40.6 & 55.4 & 0.144 & 0.148 & INS \\
\hline \multicolumn{6}{|l|}{ VIREONIDAE } \\
\hline Cyclarhis gujanensis (Gmelin, 1789) $\ddagger$ & 93.8 & 89.2 & 0.782 & 0.972 & INS \\
\hline Vireo olivaceus (Linnaeus, 1766) & 43.8 & 60 & 0.204 & 0.412 & ONI \\
\hline \multicolumn{6}{|l|}{ CORVIDAE } \\
\hline Cyanocorax criStatellus (Temminck, 1823) $\ddagger$ & 87.5 & 58.5 & - & - & ONI \\
\hline Cyanocorax chrysops (Vieillot, 1818) $\ddagger$ & 62.5 & 76.9 & - & - & ONI \\
\hline \multicolumn{6}{|l|}{ HIRUNDINIDAE } \\
\hline Pygochelidon cyanoleuca (Vieillot, 1817) & 46.9 & 36.9 & 0.046 & 0.093 & INS \\
\hline Stelgidopteryx ruficollis (Vieillot, 1817) & 46.9 & 46.2 & 0.074 & 0.042 & INS \\
\hline Progne chalybea (Gmelin, 1789) & - & 1.5 & - & - & INS \\
\hline \multicolumn{6}{|l|}{ TROGLODYTIDAE } \\
\hline Troglodytes musculus Naumann, 1823 & 46.9 & 61.5 & 0.079 & 0.102 & INS \\
\hline Cantorchilus leucotis (Lafresnaye, 1845) $* \dagger$ & 18.8 & 6.2 & 0.023 & 0.009 & INS \\
\hline \multicolumn{6}{|l|}{ DONACOBIIDAE } \\
\hline Donacobius atricapilla (Linnaeus, 1766) & - & 3.1 & - & - & INS \\
\hline \multicolumn{6}{|l|}{ POLIOPTILIDAE } \\
\hline Polioptila dumicola (Vieillot, 1817) $* \dagger$ & - & 1.5 & - & - & INS \\
\hline \multicolumn{6}{|l|}{ TURDIDAE } \\
\hline Turdus rufiventris Vieillot, 1818 & 31.3 & 61.5 & 0.097 & 0.176 & ONI \\
\hline Turdus leucomelas Vieillot, $1818 \div$ & 96.9 & 90.8 & 0.676 & 0.852 & ONI \\
\hline Turdus amaurochalinus Cabanis, 1850 & 65.6 & 64.6 & 0.194 & 0.185 & ONI \\
\hline Turdus albicollis Vieillot, $1818 *$ & - & 4.6 & - & 0.005 & ONI \\
\hline \multicolumn{6}{|l|}{ MIMIDAE } \\
\hline Mimus saturninus (Lichtenstein, 1823) & 15.6 & 24.6 & 0.019 & 0.005 & INS \\
\hline \multicolumn{6}{|l|}{ COEREBIDAE } \\
\hline Coereba flaveola (Linnaeus, 1758) & 46.9 & 49.2 & 0.102 & 0.083 & NEC \\
\hline \multicolumn{6}{|l|}{ THRAUPIDAE } \\
\hline Schistochlamys ruficapillus (Vieillot, 1817) & 31.3 & 21.5 & 0.032 & 0.005 & ONI \\
\hline
\end{tabular}

* Species that had not been recorded in EEI $(n=56)$ (review by Motta-Junior et al., 2008); $§$ Atlantic Forest endemic species $(n=16)$ (Brooks et al., 1999); $\dagger$ Species registered for the first time for the whole Itirapina region $(n=11)$ (Willis, 2003a; Willis and Oniki 2003; Motta-Junior et al., 2008); and $\ddagger$ Fragments resident species $(\mathrm{n}=23)$ (Almeida et al., 1999). 
Table 1. Continued...

\begin{tabular}{|c|c|c|c|c|c|}
\hline \multirow[t]{2}{*}{ Taxon } & \multicolumn{2}{|c|}{ FO $(\%)$} & \multicolumn{2}{|c|}{ PIA } & \multirow{2}{*}{$\begin{array}{c}\text { Trophic } \\
\text { categories }\end{array}$} \\
\hline & $\mathbf{A}$ & B & $\mathbf{A}$ & B & \\
\hline Nemosia pileata (Boddaert, 1783) & 12.5 & 9.2 & 0.009 & - & INS \\
\hline Thlypopsis sordida (d'Orbigny \& Lafresnaye, 1837) & 3.1 & 21.5 & 0.005 & 0.028 & INS \\
\hline Trichothraupis melanops (Vieillot, 1818) * & - & 4.6 & - & 0.005 & ONI \\
\hline Piranga flava (Vieillot, 1822) & 18.8 & 13.8 & 0.042 & 0.014 & ONI \\
\hline Habia rubica (Vieillot, 1817) * & - & 6.2 & - & - & INS \\
\hline Eucometis penicillata $($ Spix, 1825) $* \dagger$ & 3.1 & 10.8 & 0.005 & 0.009 & INS \\
\hline Tachyphonus coronatus (Vieillot, 1822) * $\S$ & 3.1 & 10.8 & 0.005 & 0.014 & ONI \\
\hline Ramphocelus carbo (Pallas, 1764) & 68.8 & 46.2 & 0.153 & 0.181 & ONI \\
\hline Thraupis sayaca (Linnaeus, 1766) $\ddagger$ & 87.5 & 86.2 & 0.648 & 0.625 & ONI \\
\hline Tangara cayana (Linnaeus, 1766$) \ddagger$ & 84.4 & 84.6 & 0.815 & 0.907 & ONI \\
\hline Tersina viridis (Illiger, 1811) * & - & 6.2 & - & 0.023 & ONI \\
\hline Dacnis cayana (Linnaeus, 1766) & 37.5 & 63.1 & 0.097 & 0.171 & ONI \\
\hline Hemithraupis ruficapilla (Vieillot, 1818) * $§$ & - & 1.5 & - & 0.006 & INS \\
\hline Conirostrum speciosum (Temminck, 1824) & 3.1 & 12.3 & - & 0.014 & INS \\
\hline \multicolumn{6}{|l|}{ EMBERIZIDAE } \\
\hline Zonotrichia capensis (Statius Muller, 1776) & 96.9 & 87.7 & 0.440 & 0.389 & GRA \\
\hline Ammodramus humeralis (Bosc, 1792) & 15.6 & 12.3 & 0.023 & 0.005 & GRA \\
\hline Haplospiza unicolor Cabanis, $1851 * \S \dagger$ & - & 1.5 & - & - & GRA \\
\hline Emberizoides herbicola (Vieillot, 1817) & - & 3.1 & - & 0.002 & GRA \\
\hline Volatinia jacarina (Linnaeus, 1766) & 43.8 & 50.8 & 0.125 & 0.255 & GRA \\
\hline Sporophila lineola (Linnaeus, 1758) & 12.5 & 13.8 & 0.009 & 0.014 & GRA \\
\hline Sporophila nigricollis (Vieillot, 1823) * & - & 3.1 & - & - & GRA \\
\hline Sporophila caerulescens (Vieillot, 1823) & 34.4 & 40 & 0.176 & 0.157 & GRA \\
\hline Sporophila angolensis (Linnaeus, 1766) * & 3.1 & - & - & - & GRA \\
\hline Arremon flavirostris Swainson, 1838 & 3.1 & 46.2 & 0.005 & 0.079 & INS \\
\hline Coryphospingus cucullatus (Statius Muller, 1776) $\ddagger$ & 81.3 & 64.6 & 0.366 & 0.144 & GRA \\
\hline \multicolumn{6}{|l|}{ CARDINALIDAE } \\
\hline Saltator similis d'Orbigny \& Lafresnaye, 1837 & - & 4.6 & - & - & GRA \\
\hline Saltator atricollis Vieillot, 1817 & 3.1 & - & - & - & GRA \\
\hline \multicolumn{6}{|l|}{ PARULIDAE } \\
\hline Parula pitiayumi (Vieillot, 1817) & 62.5 & 69.2 & 0.148 & 0.278 & INS \\
\hline Geothlypis aequinoctialis (Gmelin, 1789) & 50 & 27.7 & 0.074 & 0.028 & INS \\
\hline Basileuterus hypoleucus Bonaparte, $1830 \ddagger$ & 71.9 & 84.6 & 0.370 & 0.731 & INS \\
\hline Basileuterus flaveolus (Baird, 1865) $\ddagger$ & 90.6 & 89.2 & 0.505 & 0.620 & INS \\
\hline Basileuterus leucophrys Pelzeln, 1868 & 25 & - & 0.032 & - & INS \\
\hline \multicolumn{6}{|l|}{ ICTERIDAE } \\
\hline Icterus cayanensis (Linnaeus, 1766) & 3.1 & 3.1 & - & - & ONI \\
\hline Gnorimopsar chopi (Vieillot, 1819) & - & 7.7 & - & 0.005 & GRA \\
\hline Chrysomus ruficapillus (Vieillot, 1819)* & - & 9.2 & - & - & INS \\
\hline \multicolumn{6}{|l|}{ FRINGILLIDAE } \\
\hline Euphonia chlorotica (Linnaeus, 1766) $\ddagger$ & 62.5 & 78.5 & 0.157 & 0.259 & ONI \\
\hline Euphonia violacea (Linnaeus, 1758) * & 3.1 & 3.1 & 0.005 & 0.005 & ONI \\
\hline
\end{tabular}

* Species that had not been recorded in EEI ( $\mathrm{n}=56$ ) (review by Motta-Junior et al., 2008); $§$ Atlantic Forest endemic species $(\mathrm{n}=16)$ (Brooks et al., 1999); $\uparrow$ Species registered for the first time for the whole Itirapina region $(\mathrm{n}=11)$ (Willis, 2003a; Willis and Oniki 2003; Motta-Junior et al., 2008); and $\ddagger$ Fragments resident species $(\mathrm{n}=23)$ (Almeida et al., 1999).

\section{Discussion}

\subsection{Species richness}

Species richness corresponded to $27.27 \%$ of all birds of Sao Paulo State (Willis and Oniki, 2003) and 25.08\% of all birds recorded in Cerrado (Marini and Garcia, 2005). Of the 210 species recorded, six (Hylocharis sapphirina,
Melanopareia torquata, Eucometis penicillata, Sporophila angolensis, Saltator atricollis and Basileuterus leucophrys) are threatened in Sao Paulo State (São Paulo, 2008), five (M. torquata, S. atricollis, B. leucophrys, Antilophia galeata and Cyanocorax cristatellus) are endemic to the Cerrado (Silva and Bates, 2002) and sixteen are Atlantic Forest endemics (Brooks et al., 1999) (see Table 1). Two 
registered species (Chiroxiphia caudata and Habia rubica) are bioindicators (Piratelli et al., 2008). According to these authors, both species have some ecological characteristics (e.g. needs for nesting and foraging in mixed flocks) that lead them to prefer less altered areas.

Some species $(n=56)$ had never been registered in EEI (review by Motta-Junior et al., 2008) (see Table 1), but one (Leucochloris albicollis) had already been registered during a brief visit to fragment B (Willis and Oniki, 2003). This number represents a considerable increase to the bird list for the protected areas (EEI and Estação Experimental de Itirapina), now with a total of 287 species. Some species may not have been previously recorded as they are difficult to detect or even occur in the region in low relative abundance (low FO and/or low PIA values). This is the case, for example, of the five records of hummingbirds (Anthracothorax nigricollis, Thalurania glaucopis, Hylocharis sapphirina, Heliomaster squamosus, Calliphlox amethystina).

However, some of the new species recorded were found only in forested habitats, which are scarce in EEI (Granzinolli, 2009) and very important for birds in Cerrado (Piratelli and Blake, 2006). These species possibly would not be detected in more open vegetation areas, which prevail in EEI. In this group there are thirteen Atlantic Forest endemics (Brooks et al., 1999) (see Table 1). There were also, while not restricted, other species associated with forest habitats (Sick, 1997), like Eucometis penicillata, Habia rubica, Leptopogon amaurocephalus and Pachyramphus polychopterus. These results reinforce the importance of keeping forest fragments and environmental heterogeneity for the conservation of local biodiversity, especially for Cerrado. This contributes to bird conservation in a landscape scale (Bakker et al., 2002).

Within the list, there are eleven species (see Table 1) registered for the first time for the whole Itirapina region (Willis, 2003a; Willis and Oniki, 2003; Motta-Junior et al., 2008): Rostramus sociabilis, Micrastur semitorquatus, Leptotila rufaxilla, Hylocharis sapphirina, Elaenia spectabilis, Legatus leucophaius, Pachyramphus castaneus, Cantorchilus leucotis, Polioptila dumicola, Eucometis penicillata and Haplospiza unicolor. Even when a specific area is visited for a long time, recording new species is common in bird surveys, as shown by some other authors (Rodrigues et al., 2005; Motta-Junior et al., 2008). Despite the intensive sampling effort, rarefaction curves did not stabilize (see Figures 1 and 2). Jackknife estimator data indicate that bird richness is probably higher than observed for both studied areas. In light of that, new visits to the fragments are likely to allow for additional records.

\subsection{Similarities among areas}

The similarity among bird communities of fragments is probably explained by the similarities in vegetation and proximity between areas, which may favour the displacement of individuals. The matrix can also play an important role, since the occurrence of a similar matrix in distinct areas selects similar communities (Sisk et al., 1997). In addition, a considerable portion of the species common to both areas is often found in forest fragments
(Gimenes and Anjos, 2003; Antunes, 2005; Manica et al., 2010). They are less demanding species in relation to food, nesting and other surviving conditions, such as Elaenia flavogaster, Camptostoma obsoletum, Lathrotriccus euleri, Pitangus sulphuratus, Myiodynastes maculatus, Megarynchus pitangua, Tyrannus melancholicus, Cyclarhis gujanensis, Troglodytes musculus and Turdus leucomelas (Sick, 1997). The similarity of $70.95 \%$ among fragment bird communities and the probable reasons for this index, suggest that an ecological corridor to link these areas would be an important management strategy. Moreover, total fragment isolation should be avoided (Tabarelli and Gascon, 2005). In this way, corridors are proposed by some authors (Tubelis and Cavalcanti, 2000; Tubelis et al., 2004) and are, indeed, priorities for the conservation of Cerrado (Azevedo, 1998).

Lower similarity between fragments and EEI, compared to the similarity among fragments, may be related to size and vegetation differences among areas and also the presence of a large number of exclusive bird species in each one of them. The fragments have 56 exclusive species, which happens to 77 species recorded only in EEI. Belonging to this group of birds apparently sensitive to environmental changes, there is a group that includes endemic species to the Cerrado and/or threatened ones, occurring in more open vegetation forms, as Rhea americana, Culicivora caudacuta, Alectrurus tricolor and Polystictus pectoralis. Differences between study duration, frequency of visits and sampling method may also explain the disparity of results. For example Willis's survey (2004) was conducted over 21 years in contrast to the 12 months of this study.

\subsection{Frequency of occurrence}

The high percentage of species with low FO is commonly found in other studies (Almeida et al., 1999; Donatelli et al., 2007). Almeida et al. (1999) attribute the results to the occurrence of wandering, occasional or migratory species. Concerning the fragments studied, especially due to the considerable species number recorded in up to $25 \%$ of visits, this explanation can be attributed to some groups, such as diurnal raptors, represented by nine of the 11 species with low frequency in $\mathrm{A}$ and 13 of the 14 species in B. Low FO values can also be related to the reduced detection of certain species. This was true to some hummingbird species: nine of the 11 species showed low frequencies (seven species with up to $25 \%$ of frequency) in fragment $\mathrm{A}$ and the same happened with 13 of the 14 species recorded ( 9 species below $25 \%$ of frequency) in B. Species with inconspicuous vocalisations (Conirostrum speciosum, Eucometis penicillata, Piranga flava and Nemosia pileata) and/or which were recorded in low relative abundance in study areas (Penelope superciliaris, Piaya cayana and Tapera naevia) may also explain these results (Aleixo and Vielliard, 1995). Only 26 species (see Table 1) are resident (FO values higher than $75 \%$, according to Almeida et al., 1999) to the studied areas and ten of them occur in both fragments. Turdus leucomelas, Pitangus sulphuratus, Patagioenas picazuro, Cyclarhis gujanesis and Zonotrichia capensis can be included in this group because they are species adapted to disturbed 
areas and also occurred in high frequencies in other studies (Donatelli et al., 2007).

\subsection{Trophic categories}

Almost $50 \%$ of registered species were classified as predominantly insectivores and omnivores represented just over $15 \%$ of species (see Table 3 ). The percentage of birds considered frugivores was low for both fragments, corroborating studies conducted in fragments in Sao Paulo State (Motta-Junior, 1990; Manica et al., 2010). Frugivores are among the most sensitive groups to forest fragmentation (Willis, 1979) because they need large areas to reach their food needs (Pizo, 2001). The higher percentage of predominantly insectivorous birds was also recorded by Motta-Junior (1990), Donatelli et al. (2004, 2007); and Manica et al. (2010). However Motta-Junior

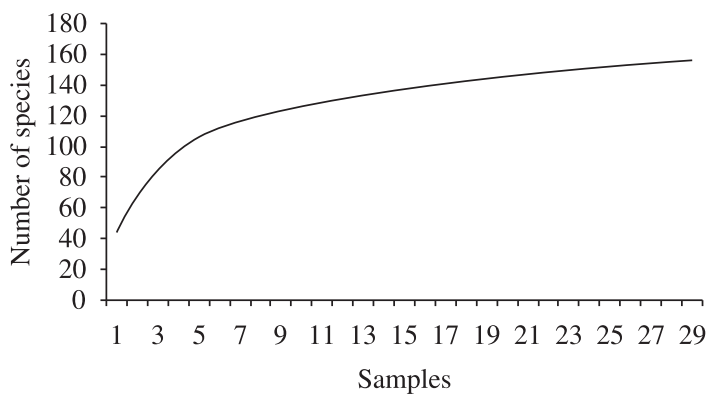

Figure 1. Rarefaction curve from fragment A during the qualitative survey conducted between October 2006 and September 2007 in Itirapina, Sao Paulo State, Brazil.
(1990) and Donatelli et al. (2007) found frugivores as the second more representative category. Omnivores were more abundant than frugivores, as observed in Motta-Junior (1990) and Manica et al. (2010) and this appears to be a common pattern in fragments (Willis, 1979). Apparently, a more varied diet (e.g. omnivory) tends to be favoured in disturbed environments.

Despite the low number of predominantly frugivores species $(\mathrm{n}=12$ ), small (Forpus xanthopterygius, Brotogeris chiriri, Antilophia galeata and Chiroxiphia caudata), medium (Aratinga leucophthalma, Aratinga aurea, Leptotila verreauxi, Leptotila rufaxilla, Patagioenas picazuro and Patagioenas cayennensis) and large (Ramphastos toco and Penelope superciliaris) size animals were found, which indicates the wide range of food items availability (Manica et al., 2010). Frugivores are important to natural

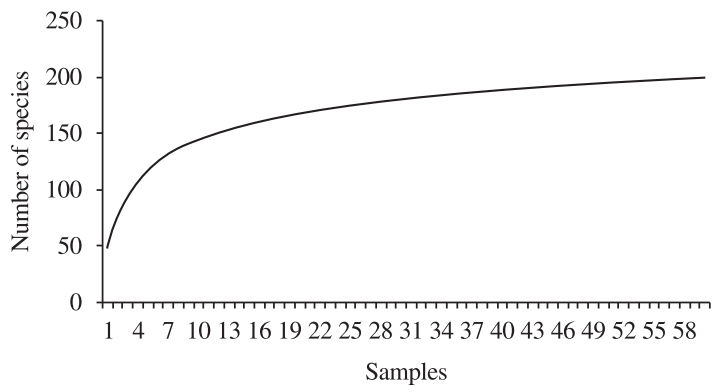

Figure 2. Rarefaction curve from fragment B during the qualitative survey conducted between October 2006 and September 2007 in Itirapina, Sao Paulo State, Brazil.

Table 2. Frequency of occurrence (FO) classes percentage of bird species in fragments A and B, Itirapina, Sao Paulo State, during 91 visits to areas in 12 months of study (October 2006 to September 2007).

\begin{tabular}{lcccccc}
\hline & & \multicolumn{4}{c}{ FO classes (\%) } & Total \\
\cline { 3 - 6 } & & $\mathbf{1 - 2 5}$ & $\mathbf{2 5 . 1 - 5 0}$ & $\mathbf{5 0 . 1 - 7 5}$ & $\mathbf{7 5 . 1 - 1 0 0}$ & \\
\hline \multirow{2}{*}{ Frag A } & species (\%) & 46.83 & 28.48 & 12.66 & 12.03 & 158 \\
& species number & 74 & 45 & 20 & 19 & \\
\multirow{2}{*}{ Frag B } & species (\%) & 52.24 & 26.37 & 14.43 & 6.97 & 201 \\
& species number & 105 & 53 & 29 & 14 & \\
\hline
\end{tabular}

Table 3. Number of species and relative percentage of nine trophic categories to the fragments studied (A and B) and analysed for both overall (total). INS - insectivores; ONI - omnivores; NEC - nectarivores; CAR - carnivores; GRA - gramnivores; PIS - piscivores; FRU - frugivores; DET - detritivores; MAL - malacophages. Categories based on Motta-Junior (1990) and Sick (1997).

\begin{tabular}{|c|c|c|c|c|c|c|c|c|c|c|c|}
\hline & & \multicolumn{10}{|c|}{ Trophic categories } \\
\hline & & INS & ONI & NEC & CAR & GRA & PIS & FRU & DET & MAL & Total \\
\hline \multirow[t]{2}{*}{ Frag A } & species & 78 & 28 & 12 & 9 & 11 & 9 & 9 & 1 & 1 & 158 \\
\hline & $\%$ & 49.37 & 17.72 & 7.59 & 5.70 & 6.96 & 5.70 & 5.70 & 0.63 & 0.63 & \\
\hline \multirow[t]{2}{*}{ Frag B } & species & 97 & 33 & 15 & 16 & 14 & 11 & 12 & 2 & 1 & 201 \\
\hline & $\%$ & 48.26 & 16.42 & 7.46 & 7.96 & 6.97 & 5.47 & 5.97 & 1.00 & 0.50 & \\
\hline \multirow[t]{2}{*}{ Total } & species & 103 & 33 & 15 & 17 & 16 & 11 & 12 & 2 & 1 & 210 \\
\hline & $\%$ & 49.05 & 15.71 & 7.14 & 8.10 & 7.62 & 5.24 & 5.71 & 0.95 & 0.48 & \\
\hline
\end{tabular}


areas because some species are related to seed dispersal (Pizo, 2001).

Although with low values, the importance of carnivores in both fragments was high when compared to other studies (Motta-Junior, 1990; Donatelli et al., 2007; Manica et al., 2010). Raptor birds have a large range (Del Hoyo et al., 1994) and probably the proximity to EEI allowed the record of most species. Therefore, these birds must use both Itirapina protected areas, which again highlight the importance of the fragments to the local avifauna. The presence of 17 carnivore species in the fragments can indicate its quality. They are top predators (Sick, 1997) and thus need a structured environment, concerning trophic chains.

Gramnivores were ranked in the fourth position, probably because both fragments have large amounts of exotic herbaceous species, used by these birds (pers.

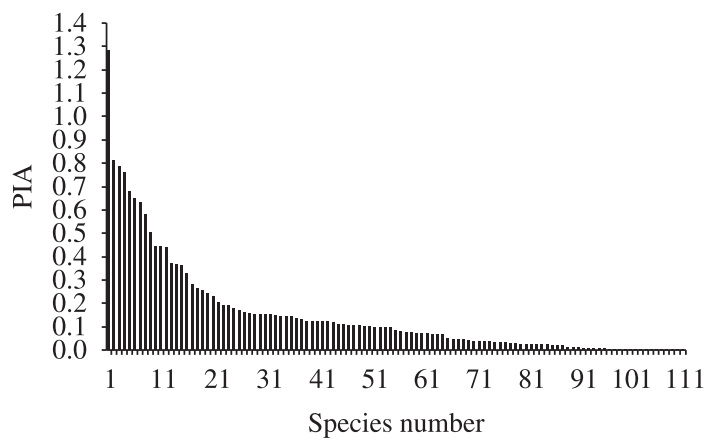

Figure 3. Ponctual Index of Abundance (PIA) in descending order of the bird species recorded during the quantitative survey conducted between October 2006 and September 2007 in fragment A in Itirapina, Sao Paulo State, Brazil. obs). Nectarivores occupied the fifth place, which can be partially attributed to the presence of Pyrostegia venusta (Ker Gawl.) Miers (Bignoniaceae), which produces flowers visited by this category (hummingbirds, mainly). However, the importance of this plant species for nectarivorous birds must be observed in other areas. Flowers of Eucalyptus spp. were also apparently important for these birds, but also requires more observations, although Willis (2002, 2003b) has already reported the importance of the resource. Nectarivores are important in natural areas because some species are flower pollinators. Impacts on pollinator populations can affect all communities' structure (Murcia, 1996).

\subsection{Ponctual index of abundance}

For the PIA values, a pattern similar to other studies that have used the same methodological standards (e.g. time in each sampling point) was observed (Vielliard and Silva, 1990; Aleixo and Vielliard, 1995; Almeida et al., 1999). The results indicated the presence of few species with high values and many species with medium to low values. Some birds had high and similar values in both fragments, such as Patagioenas picazuro, Tangara cayana, Cyclarhis gujanensis, Turdus leucomelas, Thraupis sayaca and Pitangus sulphuratus, common species in disturbed environments (Sick, 1997) and with long or constant vocalisation scope (Almeida et al., 1999). The PIA of $P$. picazuro was higher than 1.0, data also found by Almeida et al. (1999) and Donatelli et al. (2007). This species has been expanding in Sao Paulo State, aided by deforestation (Willis and Oniki, 1987) and is currently one of the most common birds in eastern Brazil (Oniki and Willis, 2000).

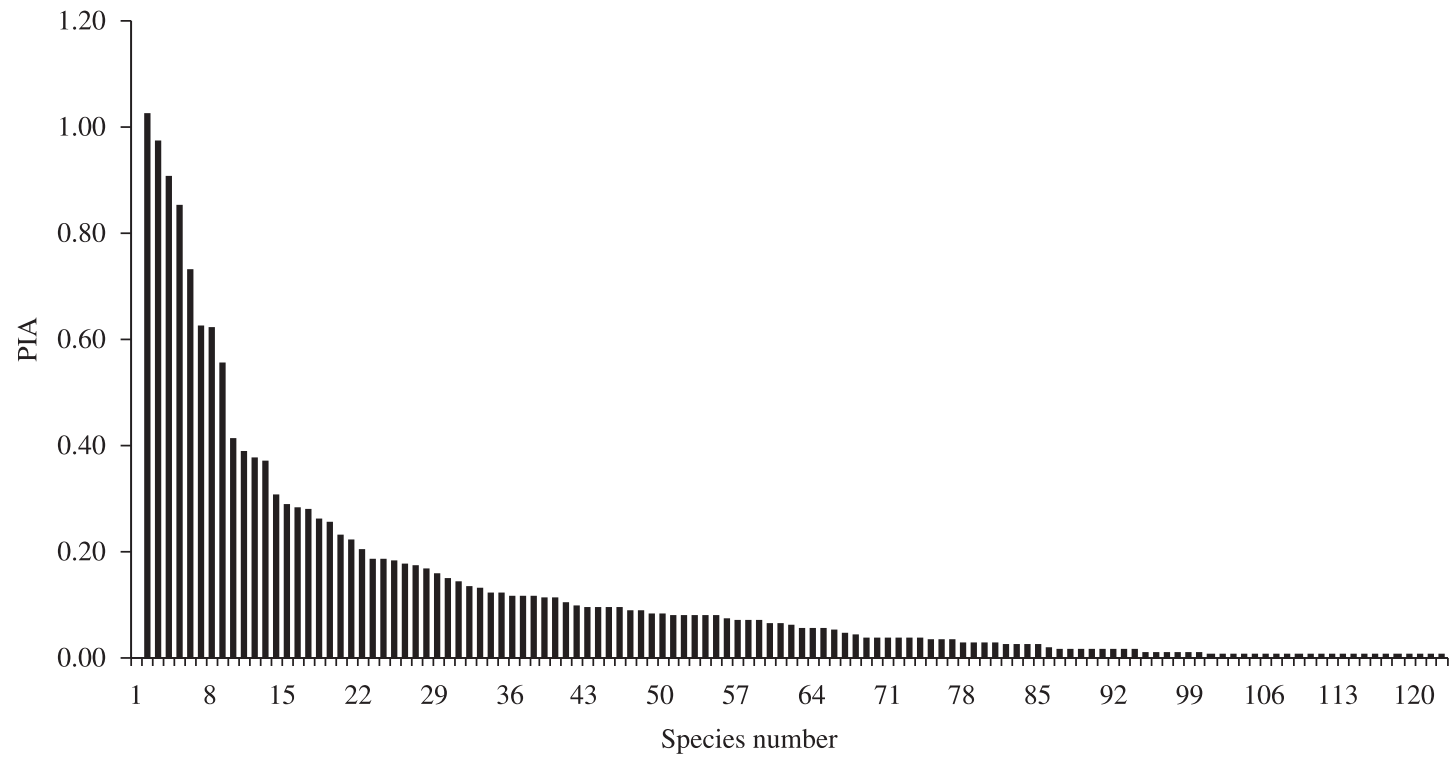

Figure 4. Ponctual Index of Abundance (PIA) in descending order of the bird species recorded during the quantitative survey conducted between October 2006 and September 2007 in fragment B in Itirapina, Sao Paulo State, Brazil. 
There is difference in PIA values of some species when the fragments are compared (see Table 1). This is the case, for example, of Tolmomyias sulphurescens, Thamnophilus caerulescens, Euphonia chlorotica, Turdus rufiventris, Conopophaga lineata and Lathrotriccus euleri, which occurred in higher relative abundance in $\mathrm{B}$, and to Coryphospingus cucullatus, Casiornis rufa, Colaptes campestris, Synallaxis frontalis and Tyrannus savanna, which occurred at higher relative abundance in A. These differences may be related to the occurrence of certain birds in forest vegetation areas, which occur in $\mathrm{B}$, and species associated with more open environments, such as those with highest relative abundance in A. This highlights the importance of keeping the Cerrado mosaic, since species are related to certain vegetation types (Boecklen, 1986).

\section{Final Considerations and Recommendations}

To improve the quality of the studied areas, this paper suggests the establishment of an ecological corridor between fragments, given the short distance that separates them, and the similarity among bird communities. It includes the use of native plant species that produce fruits. Frugivorous birds, including the largest ones, still occur within Sao Paulo State, but they need large areas to reach their food needs. So, in addition to these corridors between fragments, it could also attract frugivorous bird species, currently rare, to these fragments. Moreover, these fragments can act as a damping area to the Estação Ecológica de Itirapina, reducing the disruption in this area.

This paper also proposes discussing the withdrawal of native understory in Pinus spp. plantation, used by birds. Certain areas of forest adjacent to the fragments show native understory which is, at least apparently, important for certain bird populations. The testing of this hypothesis is therefore suggested. In case of positive results, lack of removal of native vegetation can be a simple way of management in areas of planting pine species.

\section{Conclusion}

Our results reinforce the importance of forest fragments for certain populations of birds and therefore for the conservation of birds locally. Environmental heterogeneity is an important factor for the conservation of biodiversity and therefore, is essential to the maintenance of forest fragments in areas of activities affecting the soil cover. Moreover, the results also highlight that forest fragments are important to research, since they contribute to understanding species distribution on a landscape scale, providing data for management strategies.

Acknowledgements - The authors are grateful to CNPq for the scholarship granted to the first author; Idea Wild for research equipment; the Postgraduate Programme in Ecology and Natural Resources of UFSCar for support; Sao Paulo Forestry Institute for authorising the field research, support and field assistants during the work and Matheus Reis, Mercival R. Francisco, Marcus Cianciaruso and Marco Mello for contributions to the paper.

\section{References}

ALEIXO, A. and VIELLIARD JME., 1995. Composição e dinâmica da avifauna da mata de Santa Genebra, Campinas, São Paulo, Brasil. Revista Brasileira de Zoologia, vol. 12, no. 3, p. $493-511$

ALMEIDA, MEC., VIELLIARD, JME. and DIAS, MM., 1999. Composição da avifauna em duas matas ciliares do rio Jacaré-Pepira, São Paulo, Brasil. Revista Brasileira de Zoologia, vol. 16, no. 4, p. 1087-1098.

ANTUNES, AZ., 2005. Alterações na composição da comunidade de aves ao longo do tempo em um fragmento florestal no sudeste do Brasil. Ararajuba, vol. 13, no. 1, p. 47-61.

AZEVEDO, CMA., 1998. A fragmentação e as ações governamentais para a conservação da biodiversidade. Série Técnica do Instituto de Pesquisas e Estudos Florestais, vol. 12, no. 32, p. 117-120.

BAKKER, KK., NAUGLE, DE. and HIGGINS, KF., 2002. Incorporating landscape attributes into models for migratory grassland bird conservation. Conservation Biology, vol. 16, no. 6, p. 1638-1646.

BENCKE, GA., MAURICIO, GN., DEVELEY, PF. and GOERCK, JM., 2006. Áreas importantes para a conservação das aves no Brasil. São Paulo: SAVE Brasil. 494 p. (vol. 1)

BLONDEL, J., FERRY, C. and PROCHOT, B., 1970. La méthode des indices ponctuels d'abondance (I.P.A.) ou des revelés d'avifaune par "stations d'écoute". Alauda, vol. 38, no. 1, p. 55-71.

BOECKLEN, WJ., 1986. Effects of habitat heterogeneity on the species-area relationship of forest birds. Journal of Biogeography, vol. 13 , no. 1 , p. 59-68.

BROOKS, T., TOBIAS, J. and BALMFORD, A., 1999. Deforestation and bird extinctions in the Atlantic forest. Animal Conservation, vol. 2, no. 3, p. 211-222.

COMITÊ BRASILEIRO DE REGISTROS ORNITOLÓGICOS CBRO, 2008. Lista das aves do Brasil. Rio de Janeiro: Sociedade Brasileira de Ornitologia. Available from: <http://www.cbro.org. br>. Access in: 05/12/2008.

COLWELL, RK., 2006. EstimateS: statistical estimation of species richness and shared species from samples. Version 8.0. Connecticut, USA. Available from: <http://purl.oclc.org/estimates $>$. Access in: 06/04/2009.

COUTINHO, LM., 2006. O conceito de bioma. Acta Botânica Brasileira, vol. 20, no. 1, p. 13-23.

DEL-HOYO, J., ELLIOTT, A. and SARGATAL, J., 1994. Handbook of the birds of the World. Barcelona: Lynx Edicions. 638 p. (vol. 2)

DELGADO, JM., BARBOSA, AF., SILVA, CE., ZANCHETTA, D., SILVA, DA., GIANNOTI, D., PINHEIRO, GS., DUTRA-LUTGENS, H., FACHIN, HC., MOTA, IS., LOBO, MT., NEGREIROS, OC. and ANDRADE, WJ., 2004. Plano de manejo integrado das unidades de Itirapina - SP. São Paulo: Instituto Florestal.

DONATELLI, RJ., COSTA, TVV. and FERREIRA, CD., 2004. Dinâmica da avifauna em fragmento de mata na Fazenda Rio Claro, Lençóis Paulista, São Paulo, Brasil. Revista Brasileira de Zoologia, vol. 21, no. 1, p. 97-114.

DONATELLI, RJ., FERREIRA, CD., DALBETO, AC. and POSSO, SR., 2007. Análise comparativa da assembléia de aves em dois 
remanescentes florestais no interior de São Paulo, Brasil. Revista Brasileira de Zoologia, vol. 24, no. 2, p. 362-375.

DURIGAN, G., SIQUEIRA, MF. and FRANCO, GADC., 2007. Threats to the Cerrado remnants of the State of São Paulo, Brazil. Scientia Agricola, vol. 64, no. 4, p. 355-363.

GIMENES, MR. and ANJOS, L., 2003. Efeitos da fragmentação florestal sobre as comunidades de aves. Acta Scientiarum, vol. 25, no. 2, p. 391-402.

GRANZINOLLI, MA., 2009. Levantamento, área de vida, uso e seleção de hábitat de Falconiformes na região central do Estado de São Paulo. São Paulo: Universidade de São Paulo. [Tese de Doutorado]

HAMMER, Ø., HARPER, DAT. and RYAN, PD., 2001. PAST: Palaeontological Statistics software package for education and data analysis. Version 1.91. Oslo, Norway. Palaeontologia Eletronica, vol. 4, no. 1, 9 p. Available from: <http://folk.uio.no/ohammer/ past>. Access in: 06/04/2009.

HELTSHE, J. and FORRESTER, NE., 1983. Estimating species richness using the jackknife procedure. Biometrics, vol. 39, no. 1 , p. 1-11.

INSTITUTO BRASILEIRO DO MEIO AMBIENTE E DOS RECURSOS NATURAIS RENOVÁVEIS - IBAMA, 2008. Lista das espécies da fauna ameaçadas de extinção. Brasília.

INTERNATIONAL UNION FOR CONSERVATION OF NATURE AND NATURAL RESOURCES - IUCN, 2008. IUCN Red List of Threatened Species. Cambridge, UK. Available from: <http:// www.iucnredlist.org >. Access in: 11/01/2009.

KLINK, CA. and MACHADO, RB., 2005. Conservation of the brazilian Cerrado. Conservation Biology, vol. 19, no. 3, p. $707-713$

KREBS, CJ., 1999. Ecological methodology. California: Ed. Addison-Welsey. $620 \mathrm{p}$.

MACHADO, RB., RAMOS NETO, MB., PEREIRA, PGP., CALDAS, EF., GONÇALVES, DA., SANTOS, NS., TABOR, K. and STEININGER, M., 2004. Estimativas de perda da área do Cerrado brasileiro. Brasília: Conservação Internacional. 26 p.

MANICA, LT., TELLES, M. and DIAS, MM., 2010. Bird richness and composition in a cerrado fragment in the State of São Paulo. Brazilian Journal of Biology, vol. 70, no. 2, p. 243-254.

MARINI, MÂ., 2001. Effects of forest fragmentation on birds of the Cerrado region, Brazil. Bird Conservation International, vol. 11, p. 11-23.

MARINI, MÂ. and GARCIA, FI., 2005. Bird conservation in Brazil. Conservation Biology, vol. 19, no. 3, p. 665-671.

MOTTA-JUNIOR, JC., 1990. Estrutura trófica e composição das avifaunas de três habitats terrestres na Região Central de Estado de São Paulo. Ararajuba, vol. 1, p. 65-71.

MOTTA-JUNIOR, JC., GRANZINOLLI, MAM. and DEVELEY, PF., 2008. Aves da Estação Ecológica de Itirapina, Estado de São Paulo, Brasil. Biota Neotropica, vol. 8, no. 3, p. 207-227.

MURCIA, C., 1996. Forest fragmentation and the pollination of neotropical plants. In SCHELHAS, J. and GREENBERG, RS. (Eds.). Forest Patches in Tropical Landscapes. Washington: Island Press. p. 19-36.
MYERS, N., MITTERMEIER, RA., MITTERMEIER, CG., FONSECA, GAB. and KENT, J., 2000. Biodiversity hotspots for conservation priorities. Nature, vol. 403, no. 6772, p. 853-858.

NAEVE, HM., NORTON, TW. and NIX, HA., 1996. Biological inventory for conservation evaluation II. Composition, functional relationships and spatial prediction of bird assemblages in southern Australia. Forest Ecology and Management, vol. 85, no. 1-3, p. 123-148.

ONIKI, Y. and WILLIS, EO., 2000. Nesting behavior of the picazuro pigeon, Columba picazuro (Columbidae, aves). Revista Brasileira de Biologia = Brazilian Journal of Biology, vol. 60, no. 4, p. 663-665.

PALMER, GC., FITZSIMONS, JA., ANTOS, MJ. and WHITE, JG., 2008. Determinants of native avian richness in suburban remnant vegetation: implications for conservation planning. Biological Conservation, vol. 141, no. 9, p. 2329-2341.

PIRATELLI, A. and BLAKE, JG., 2006. Bird communities of the southeastern cerrado region, Brazil. Ornitologia Neotropical, vol. 17, no. 3, p. 213-225.

PIRATELLI, A., SOUSA, SD., CORRÊA, JS., ANDRADE, VA., RIBEIRO, RY., AVELAR, LH. and OLIVEIRA, EF., 2008. Searching for bioindicators of forest fragmentation: passerine birds in the Atlantic forest of southeastern Brazil. Brazilian Journal of Biology, vol. 68, no. 2, p. 259-268.

PIZO, MA., 2001. A conservação das aves frugívoras. In ALBUQUERQUE, JLB., CÂNDIDO, JF., STRAUBE, FC. and ROOS, AL. (Eds.). Ornitologia e conservação: da ciência às estratégias. Tubarão, SC: Ed. Unisul. p. 49-59.

RODRIGUES, M., CARRARA, LA., FARIA, LP. and GOMES, HB., 2005. Aves do Parque Nacional da Serra do Cipó: o Vale do Rio Cipó, Minas Gerais, Brasil. Revista Brasileira de Zoologia, vol. 22 , no. 2, p. 326-338.

SÃO PAULO. Secretaria do Meio Ambiente, 1999. Conhecer para conservar: as unidades de conservação do Estado de São Paulo. São Paulo: Série Probio. 115 p.

SÃO PAULO, 2008. Decreto no. 53.494, de 02 de outubro de 2008. Declara as espécies da fauna silvestre ameaçadas, as quase ameaçadas, as colapsadas, sobrexplotadas, ameaçadas de sobrexplotação e com dados insuficientes para avaliação no Estado de São Paulo e dá providências correlatas. Diário Oficial do Estado de São Paulo, Poder Executivo, São Paulo, 03 out. 2008

SICK, H., 1997. Ornitologia brasileira. Rio de Janeiro: Nova Fronteira. $862 \mathrm{p}$.

SILVA, JMC. and BATES, JM., 2002. Biogeografic patterns and conservation in the South American Cerrado: a tropical savanna hotspot. BioScience, vol. 52, no. 3, p. 225-233.

SISK, TD., HADDAD, N. and EHRLICH, PR., 1997. Bird assemblages in patch woodlands: modeling the effect of edge and matrix habitats. Ecological Applications, vol. 7, no. 4, p. 1170-1180.

TABARELLI, M. and GASCON, C., 2005. Lessons from fragmentation research: improving management and policy guidelines for biodiversity conservation. Conservation Biology, vol. 19 , no. 3 , p. $734-739$.

TUBELIS, DP. and CAVALCANTI, RB., 2000. A comparison of bird communities in natural and disturbed non-wetland open habitats in the Cerrado's central region, Brazil. Bird Conservation International, vol. 10, no. 4, p. 331-350. 
TUBELIS, DP., COWLING, A. and DONNELLY, C., 2004. Landscape supplementation in adjacent savannas and its implications for the design of corridors for forest birds in the central Cerrado, Brazil. Biological Conservation, vol. 118, no. 3, p. 353-364.

VIELLIARD, JME., 2000. Bird community as an indicator of biodiversity: results from quantitative surveys in Brazil. Anais $d a$ Academia Brasileira de Ciências, vol. 72, no. 3, p. 323-330.

VIELLIARD, JME. and SILVA, WR., 1990. Nova metodologia de levantamento quantitativo e primeiros resultados no interior de São Paulo. In Anais do IV Encontro Nacional de Anilhadores de Aves. Pernambuco: Universidade de Pernambuco. p. 117-151.

WILLIS, EO., 1979. The composition of avian communities in remanescent woodlots in southern Brazil. Papéis Avulsos de Zoologia, vol. 33, no. 1, p. 1-25.

-, 2002. Birds at Eucalyptus and other flowers in Southern Brazil: a review. Ararajuba, vol. 10, no. 1, p. 43-66.
-, 2003a. Birds of a Neotropical woodlot after fire. Ornitologia Neotropical, vol. 14, no. 2, p. 233-246.

-, 2003b. Birds of a Eucalyptus woodlot in interior São Paulo. Brazilian Journal of Biology, vol. 63, no. 1, p. 141-158.

-, 2004. Birds of a habitat spectrum in the Itirapina savanna, São Paulo, Brazil. Brazilian Journal of Biology, vol. 64, no. 4, p. 901-910.

WILLIS, EO. and ONIKI, Y., 1987. Invasion of deforested regions of São Paulo State by the picazuro pigeon, Columba picazuro Temminck 1813. Ciência e Cultura, vol. 39, no. 11, p. 1064-1065.

-, 2003. Aves do Estado de São Paulo. Rio Claro: Editora Divisa. $398 \mathrm{p}$.

ZANCHETTA, D., 2006. Plano de manejo das unidades integradas de Itirapina, SP. 1 rev. Itirapina, São Paulo: Instituto Florestal de São Paulo. 